\title{
Capítulo IV - Situações especiais
}

\section{Asma no IDOSO}

A asma é subdiagnosticada no idoso por várias razões ${ }^{(1-3)}$ :

- Menor percepção da dispnéia

- Interpretação da dispnéia como uma conseqüência natural da idade

- Doenças associadas (cardiovasculares, hipotiroidismo)

- Dificuldade de comprovação objetiva da obstrução das vias aéreas

As etapas do tratamento não diferem das dos indivíduos de outras faixas etárias, mas alguns aspectos merecem ser enfatizados(3-7):

- possíveis co-morbidades podem estar presentes

- os $\beta_{2}$-agonistas podem provocar tremores ou agravar tremores preexistentes

- os $\beta_{2}$-agonistas podem ter efeitos menos expressivos, já que pode haver disfunção de $\beta$-receptores

- por sua maior segurança os anticolinérgicos devem ser indicados quando for necessária medicação broncodilatadora contínua

- a vacinação anual antiinfluenza e a antipneumocócica a cada 5-7 anos devem ser indicadas

- a técnica de utilização de medicações inalatórias deve ser revisada regularmente e a utilização de espaçadores para o uso dos aerossóis dosimetrados deve ser estimulada quando inaladores de pó não puderem ser utilizados

- é fundamental verificar a adesão, que pode não ser adequada devido à prescrição de vários medicamentos, declínio cognitivo com perda de memória, limitações físicas, dificuldade na compra por questões financeiras ou apatia.

\section{Asma na GRAVIDEZ}

A gravidez tem um efeito variável sobre o curso da asma, podendo permanecer estável, piorar ou melhorar, com retorno ao estado anterior à gravidez em cerca de três meses após o parto(8).

Os sintomas geralmente melhoram durante as últimas quatro semanas da gravidez e o parto não costuma associar-se com piora da asma. 0 curso da asma em sucessivas gestações costuma ser semelhante em cada paciente ${ }^{(9)}$.

O manejo difere muito pouco daquele preconizado para não-grávidas. 0 subtratamento resulta em maior risco para a mãe e para o feto do que o uso de quaisquer drogas necessárias para o controle da doença ${ }^{(10-12)}$. 0 baixo risco de malformações congênitas associadas às medicações usualmente utilizadas no tratamento da asma está documentado ${ }^{(13,14)}$.

Pacientes com asma malcontrolada devem ser cuidadosamente monitorizadas quanto ao retardo de crescimento intra-uterino do feto e pré-eclâmpsia ${ }^{(13,15)}$. Monitorização fetal intensiva é essencial em mulheres com asma aguda durante a gravidez. Algumas medicações potencialmente usadas para indicações obstétricas em pacientes com asma devem ser evitadas pela possibilidade de broncoespasmo. Estas incluem prostaglandina $\mathrm{F}_{2 \alpha^{\prime}}$ ergonovina e agentes antiinflamatórios não esteróides (pacientes sensíveis). Ocitocina é a droga de escolha para indução do parto. Crises de asma durante o parto devem ser tratadas de maneira usual.

A categorização das drogas na gravidez encontra-se no Quadro 1.

O Quadro 2 mostra as drogas utilizadas para asma na gravidez com as respectivas categorias propostas pelo FDA.

\section{Asma e cirurgia}

Os asmáticos apresentam maior risco de complicações pulmonares pós-operatórias e o broncoespasmo intraoperatório deve ser encarado como uma complicação com potencial risco para a vida(16,17). Tem sido sugerido que pacientes com história de atopia apresentam maior risco de reações de hipersensibilidade imediata (rinite, asma, anafilaxia, etc.) ou reações anafilactóides (pseudo-alérgicas) durante 0 ato cirúrgico ou testes diagnósticos (contrastes radiológicos). Mais recentemente, a hipersensibilidade ao látex das luvas cirúrgicas ou aos materiais utilizados em ambiente hospitalar, tais como cateteres, sondas e cânulas, tem sido implicada em casos de anafilaxia durante 0 ato cirúrgico.

Durante a anestesia, a intubação traqueal constitui-se no mais vigoroso estímulo para o aparecimento do broncoespasmo. 0 uso do $\beta_{2}$ de ação curta pode prevenir 0 aumento da resistência do sistema respiratório em situações anestésicas especiais(18).

O uso do corticosteróide inalatório reduz a HR e previne as exacerbações durante a cirurgia. Portanto, é fundamental alcançar o controle adequado da asma antes de qualquer procedimento e ter uma conduta segura no perioperatório ${ }^{(19)}$.

\section{Asma no lactente}

Em lactentes susceptíveis, a presença de atopia predispõe à sensibilização por alérgenos ambientais ou irritan- 


\section{QUADRO 1 \\ Drogas na gravidez - Classificação do FD A}

Categoria

Interpretação

A Estudos controlados mostram risco ausente

Estudos bem controlados, adequados, em mulheres grávidas não demonstram risco para o feto.

B Nenhuma evidência de risco em humanos

Achados em animais mostraram risco, mas em humanos não ou, se estudos humanos adequados não foram feitos, os achados em animais foram negativos.

C Risco não pode ser excluído

$\mathrm{N}$ ão existem estudos positivos em humanos e em animais para risco fetal ou simplesmente inexistem estudos. Contudo, os benefícios potenciais justificam o risco potencial.

D Evidência positiva de risco

Dados de investigação ou após liberação no mercado mostram risco para o feto. M esmo assim, os benefícios potenciais podem sobrepujar o risco.

$X \quad$ Contra-indicado na gravidez

Estudos em animais e humanos, ou relatados de investigação ou após liberação no mercado, mostraram risco fetal, que claramente é maior que os benefícios potenciais.

\begin{tabular}{|c|c|c|}
\hline \multicolumn{3}{|c|}{ Q U ADRO 2 } \\
\hline D rogas antiasmáticas na gravidez \\
\hline \multirow{2}{*}{$\beta$-agonistas } & Droga específica & Categoria FD A \\
& Salbutamol & C \\
& Epinefrina & C \\
Metilxantinas & Salmeterol & C \\
Anticolinérgicos & Terbutalina & B \\
Corticosteróides & Teofilina & C \\
& Ipratrópio & B \\
& Prednisona & Não classificada \\
& Budesonida & Não classificada \\
& Beclometasona & C \\
Cromonas & Triamcinolona & C \\
& Flunisolida & C \\
Antileucotrienos & Fluticasona & B \\
& Cromoglicato de sódio & B \\
& Nedocromil & B \\
& Zafirlucaste & B \\
\hline
\end{tabular}


tes e, desse modo, a quadros recorrentes de sibilância, sendo a exposição precoce aos ácaros domésticos, fungos e antígenos derivados de animais muito importante para a sensibilização( ${ }^{(20)}$.

0 desenvolvimento de atopia em fase precoce da vida parece relacionar-se à presença de $\mathrm{HR}$ das vias aéreas em idade posterior. Por outro lado, dados conflitantes apontam para o fato de que a asma iniciada nos dois primeiros anos de vida pode resultar em função pulmonar reduzida na idade adulta, indicando possível ação deletéria da asma no desenvolvimento da função pulmonar ${ }^{(21,22)}$.

0 Quadro 3 define risco de asma em lactentes. Aqueles que apresentam dois critérios maiores, sendo um deles o número 1 ou 2, ou aqueles que apresentarem dois critérios maiores e dois menores, devem ser considerados de alto risco para sibilância persistente e diagnóstico de asma(23).

0 manejo do lactente com sibilância tem sido muito discutido, especialmente no que diz respeito ao uso dos corticosteróides inalatórios. É inequívoco o benefício desses medicamentos no tratamento da asma em todas as idades, mas há alguns argumentos que costumam ser debatidos quando se trata de lactentes ${ }^{(24-26)}$.

Argumentos contra o uso de corticosteróides inalatórios em lactentes e pré-escolares:

- Nem sempre é possível identificar precocemente os asmáticos, e as crises de obstrução brônquica são devidas a várias doenças com diferentes fisiopatologias, vias aéreas estreitas e infecções virais, muitas vezes constituindo uma condição transitória e que só necessita de medicação sintomática.

- Cerca de 80\% dos lactentes chiadores não continuarão a apresentar crises de obstrução brônquica na infância e adolescência. Com base em critérios clínicos, é muito difícil predizer se um lactente chiador será asmático no futuro.
- Nos lactentes chiadores, a maioria dos episódios de obstrução brônquica é de origem viral e, quando não há atopia associada, eles permanecem assintomáticos entre as crises.

- Recém-nascidos com baixo peso para a idade gestacional e filhos de mães fumantes têm função pulmonar reduzida, mas melhoram com o crescimento pulmonar.

- Existem poucos estudos em animais sobre o uso de corticosteróide inalatório e crescimento pulmonar e também poucos trabalhos sobre 0 uso de corticosteróide inalatório em lactentes. As conclusões obtidas, até o presente momento, mostram que há risco potencial de o uso de corticosteróide inalatório afetar o crescimento pulmonar, principalmente nos dois primeiros meses de vida e que 0 impacto da perda de fibras elásticas, na velhice, deverá ser maior na população que utilizou corticosteróide inalatório precocemente.

\section{Asma induzida Pelo exercício}

Os termos asma induzida pelo exercício (AIE) e broncoconstrição induzida pelo exercício (BIE) têm sido usados como sinônimos para expressar a resposta broncoespástica que alguns indivíduos apresentam ao se exercitar. Quarenta a noventa por cento dos asmáticos e $40 \%$ dos pacientes com rinite alérgica apresentam $\mathrm{BIE}^{(27,28)}$.

A patogênese da BIE está associada ao fluxo de calor e água da mucosa brônquica em direção à luz do brônquio, com o objetivo de condicionar grandes volumes de ar que chegam ao trato respiratório inferior ${ }^{(28)}$.

0 exercício é o único precipitante natural da asma que induz taquifilaxia. Cerca de $45 \%$ dos pacientes com AIE apresentam um período refratário característico, durante o qual a manutenção do exercício não causa broncoespasmo. Quando o exercício é repetido após intervalos de 30 a 90 minutos, verifica-se que a broncoconstrição diminui ou não ocorre ${ }^{(27,29)}$. A presença de um período refratário parece ser independente do grau de obstrução

QUADRO 3

Critérios para diagnóstico de asma

Critérios maiores

1. H ospitalização por bronquiolite ou sibilância grave

2. Pelo menos três episódios de sibilância durante os primeiros seis meses de vida

3. História parental de asma

4. Dermatite atópica

\section{Critérios menores}

1. Rinorréia não associada a resfriado

2. Sibilância não associada a resfriado

3. Eosinofilia maior ou igual a $5 \%$

4. Sexo masculino 
provocado pelo primeiro teste e inversamente relacionada ao tempo que separa as duas provocações consecutivas pelo exercício.

Outro aspecto controverso relacionado à BIE é a existência ou não de uma resposta tardia ao exercício (três a 12 horas após). Alguns estudos observaram uma resposta tardia ao exercício, com uma prevalência variando em torno de $10 \%$ a $89 \%(27,28,30)$. O diagnóstico de BIE é feito através da história clínica e do teste de desencadeamento com exercício monitorado pela função pulmonar.

A obstrução da via aérea costuma iniciar logo após o exercício, atingindo seu pico entre cinco e 10 minutos, após o que há remissão espontânea do broncoespasmo, com melhora total da função pulmonar em torno de 30 a 60 minutos. Os pacientes em crise de asma precipitada pelo exercício apresentam os mesmos sintomas observados em crises desencadeadas por outros estímulos ${ }^{(27,28)}$.

A presença de BIE pode ser demonstrada em nível laboratorial através da queda de $10 \%$ do volume expiratório forçado no primeiro segundo $\left(\mathrm{VEF}_{1}\right)$ em relação ao $\mathrm{VEF}_{1}$ basal, pré-exercício. Alguns autores consideram que uma queda de $15 \%$ do $\mathrm{VEF}_{1}$ define um diagnóstico mais preciso(31,32).

Alternativamente, pode ser usado o pico de fluxo ou ainda a resistência e a condutância específica das vias aéreas. Beck et al. demonstraram que alterações na resistência pulmonar e no $\mathrm{VEF}_{1}$ produzem índices equiva-

\section{ReferênCIAS}

1. Peat JK, Woolcock AJ, Cullen K. Rate of decline of lung function in subjects with asthma. Eur J Respir Dis 1987;70:171.

2. Sherman $C B, H$ ubert $M$, Fogel $B S$. Unrecognized respiratory disease in the elderly. Am Respir Dis 1992;145:763.

3. Sherman CB. Late-onset asthma: making the diagnosis, choosing drug therapy. Geriatrics 1995;50:24-33.

4. Armitage JM, Williams SJ. Inhaler technique in the elderly. Age Ageing 1998;17:275-8.

5. Burrows B, Barbee RA, Cline MG, Knudson RJ, Lebowitz MD. Characteristics of asthma among elderly adults in a sample of the general population. Chest 1991;100:935-42.

6. Chapman K, Love L, Brubaker H. A comparison of breath-actuated and conventional MDI inhalation techniques in elderly subjects. Chest 1993;104:1332-7.

7. Steward R, Caranasos G. Adesão a medicamentos em idosos. Clin Med Am Norte 1967;6:1710.

8. Gluck JC, G kuck PA. The effects of pregnancy on asthma: a prospective study. Ann Allergy 1976;37:164-8.

9. Tan KS, Thomson NC. Asthma and pregnancy. Am J Med 2000;109: 727-33.

10. Hasbargen U, Reber D, Versmold H, Schulze A. Growth and development of children to 4 years of age after repeated antenatal steroid administration. Eur J Pediatr 2001;160:552-5.

11. Olesen C, Thrane N, Nielsen GL, Sorensen HT, Olsen J, Euro MAP Group. A population-based prescription study of asthma drugs during pregnancy: changing the intensity of asthma therapy and perinatal outcomes. Respiration 2001;68:256-61. lentes de variação da função pulmonar induzida por exercício ${ }^{(33)}$

O objetivo do tratamento de pacientes com BIE é a profilaxia.

Episódios de BIE podem ser atenuados com a elaboração de uma escala personalizada de aquecimento antes da realização de um exercício físico vigoroso(34).

0 controle mais efetivo, entretanto, é obtido com medicações. 0 primeiro passo no manejo da BIE deve ser o controle da asma subjacente. 0 tratamento regular da asma com corticóide inalatório costuma reduzir a magnitude da BIE em $50 \%{ }^{(35)}$.

Contudo, muitos pacientes necessitam tratamento adicional.

Os $\beta_{2}$-agonistas inalatórios de curta duração, se utilizados 15 a 30 minutos antes do exercício, inibem a BIE. A duração desse efeito protetor é de quatro horas.

Os antileucotrienos também protegem de forma satisfatória a BIE, e seu uso regular parece não estar associado com tolerância e redução de seu efeito protetor $r^{(36,37)}$.

Os $\beta_{2}$-agonistas inalatórios de longa duração podem ser usados para impedir a BIE, porém a duração desse efeito protetor pode diminuir com o uso continuado(36,38).

As cromonas inalatórias atenuam a BIE em alguns pacientes. Seu efeito protetor usualmente dura em torno de duas a três horas ${ }^{(39)}$.

12. Spector SL. Safety of antileukotriene agents in asthma management. Ann Allergy Asthma Immunol 2001;86:18-23.

13. J adad AR, Sigouin C, Mohide PT, Levine M, Fuentes M. Risk of congenital malformations associated with treatment of asthma during early pregnancy. Lancet 2000;355:119.

14. Lehrer S, Stone J, Lapinski R, Lockwood CJ, Schachter BS, Berkowitz $R$, et al. Association between pregnancy-induced hypertension and asthma during pregnancy. Am J Obstet Gynecol 1993;168: 1463-6.

15. Clifton VL, Giles WV, Smith R, Bisits AT, Hempenstall PA, Kessel CG, et al. Alterations of placental vascular function in asthmatic pregnancies. Am J Respir Crit Care Med 2001;164:546-53.

16. Brandus $V$, Wolff $W$, J offe $S$, Benoit $C$. Bronchial spasm during general anesthesia. Can Anaesth Soc J 1970;17:269-74.

17. Keenan RL, Boyan CP. Cardiac arrest due to anesthesia. JAMA 1985; $253: 2373$.

18. Scalfaro P, Sly PD, Sims C, Habre W. Salbutamol prevents the increase of respiratory resistance causes by tracheal intubation during seflurane anesthesia in asthmatic children. Anesth Analg 2001;93:898902.

19. Mitsuda K, Shimoda T, Kukushima C, Obase $Y$, Ayabe H, Matsuse H, et al. Preoperative steroid therapy inhibits cytokine production in the lung parenchyma in asthmatic patients. Chest 2001;120:1175-83.

20. Clough JB, William JD, Holgate ST. Effect of atopy on the natural history of symptoms, peak flow, and bronchial responsiveness in 7 and 8-year old children with cough and wheeze. Am J Respir Crit Care Med 1991;143:755-60. 
21. Sherrill D. The effects of airway hyperresponsiveness, wheezing, and atopy on longitudinal pulmonary function in children: a 6-year followup study. Pediatr Pulmonol 1992;13:78-85.

22. Van Asperen PP, Kamp AS, Mukhi A. Atopy in infancy predicts the severity of bronchial hyperresponsiveness in later childhood. J Allergy Clin Immunol 1990;85:790-5.

23. Martinez FD. Recognizing early asthma. Allergy 1999;54:24-8.

24. Landau LI. Inhaled corticosteroids in childhood asthma. Pediatr Pulmonol 1999;27:365-8.

25. Pedersen S, Warner J O. Early use of inhaled steroids in children. Clin Exp Allergy 1997;27:995-1006.

26. Pedersen S, O'Byrne PA. A comparation of the efficacy and safety of inhaled corticosteroids in asthma. Allergy 1997;52(Suppl 390):1-34.

27. Kumar A, Busse WW. Recognizing and controlling exercise-induced asthma. J Respir Dis 1995;16:1087-96.

28. MCFadden ER, Gilbert IA. Exercise-induced asthma. N Engl J Med 1994;330:1362-7.

29. Wright LA. Nocturnal asthma and exercise-induced bronchospasm. Postgrad Med J 1995;97:83-90.

30. Peroni BA. Exercise-induced asthma: is there a space for late-phase reactions? Eur J Respir Dis 1996;9:1335-8.

31. ATS. ATS Guidelines for methacholine and exercise challenge testing. Am J Respir Crit Care Med 1999;161:309-29.
32. National Health LaBI. Global Strategy for Asthma Management and Prevention. NHLBI/WHO Workshop 1993;95:1-167.

33. Beck KC, Hyatt RE, Mpougas P, Scanlon PD. Evaluation of pulmonary resistance and maximal expiratory flow measurements during exercise in humans. J Appl Physiol 1999;86:1388-95.

34. Bisschop C, Guenard H, Desnot O, Vergeret J. Reduction of exerciseinduced asthma in children by short, repeated warm-ups. Br J Sports Med 1999;33:100-4.

35. Inman MD. Management of exercise-induced bronchoconstriction. Can Respir J 1999;6:345-54.

36. Edelman J M, Turpin JA, Bronsky EA, Grossman J, Kemp JP, Ghannam $A F$, et al. Oral Montelukast compared with inhaled salmeterol to prevent exercise-induced bronchoconstriction. A randomized, doubleblind trial. Ann Intern Med 2000;132:97-104.

37. Leff J A, Busse WW, Pearlman D, Bronsky EA, Kemp J, Hendeles L, et al. Montelukast, a leukotriene-receptor antagonist, for the treatment of mild asthma and exercise-induced bronchoconstriction. N Engl J Med 1998;339:147-52.

38. Gronnerod TA, Von Berg A, Schwabe G, Soliman S. Formoterol via turbuhaler gave better protection than terbutaline against repeated exercise challenge for up to 12 hours in children and adolescents. Respir Med 2000;94:661-7.

39. Kelly KD, Spooner $\mathrm{CH}$, Rowe $\mathrm{BH}$. Nedocromil sodium versus sodium cromoglycate in treatment of exercise-induced bronchoconstriction: a systematic review. Eur J Respir Dis 2001;17:39-45. 